Research Paper

\title{
Competitive Performance of Carbon "Quantum" Dots in Optical Bioimaging
}

\author{
Li Cao, Sheng-Tao Yang, Xin Wang, Pengju G. Luo, Jia-Hui Liu, Sushant Sahu, Yamin Liu, Ya-Ping Sun $\bowtie$
}

Department of Chemistry and Laboratory for Emerging Materials and Technology, Clemson University, Clemson, South Carolina 29634-0973, USA.

Corresponding author: Tel: 864-656-5026, Fax: 864-656-6613, E-mail: syaping@clemson.edu

(c) Ivyspring International Publisher. This is an open-access article distributed under the terms of the Creative Commons License (http://creativecommons.org/ licenses/by-nc-nd/3.0/). Reproduction is permitted for personal, noncommercial use, provided that the article is in whole, unmodified, and properly cited.

Received: 2011.12.04; Accepted: 2012.01.01; Published: 2012.03.07

\begin{abstract}
Carbon-based "quantum" dots or carbon dots are surface-functionalized small carbon nanoparticles. For bright fluorescence emissions, the carbon nanoparticles may be surface-doped with an inorganic salt and then the same organic functionalization. In this study, carbon dots without and with the $\mathrm{ZnS}$ doping were prepared, followed by gel-column fractionation to harvest dots of $40 \%$ and $60 \%$ in fluorescence quantum yields, respectively. These highly fluorescent carbon dots were evaluated for optical imaging in mice, from which bright fluorescence images were obtained. Of particular interest was the observed competitive performance of the carbon dots in vivo to that of the well-established CdSe/ZnS QDs. The results suggest that carbon dots may be further developed into a new class of high-performance yet nontoxic contrast agents for optical bioimaging.
\end{abstract}

Key words: Carbon dots, fluorescence, optical bioimaging, quantum dots.

\section{Introduction}

Small carbon nanoparticles have been shown as versatile precursors for brightly fluorescent nano-dots [1-15], with the observed optical properties in solution phenomenologically similar to and performance-wise comparable with those of the well-established semiconductor quantum dots (QDs) [9, 16-19]. Structurally, the fluorescent carbon-based "quantum" dots (or often referred to as carbon dots) are small carbon nanoparticles with relatively simple particle surface functionalization $[1,2,7,10,11]$. Carbon dots with oligomeric polyethylene glycol diamine ( $\mathrm{PEG}_{1500 \mathrm{~N}}$, Scheme 1/Figure S1) [20] as the surface functionalization agent were shown to be nontoxic [21], amenable to fluorescence bioimaging applications in vitro and in vivo $[10,11,22,23]$. More recently, highly fluorescent carbon dots with emission quantum yields more than $50 \%$ were obtained by surface-doping the core carbon nanoparticles with an inorganic salt such as $\mathrm{ZnS}$ (Scheme 1/Figure S1) or $\mathrm{TiO}_{2}[20,24]$, and/or by ap- plying a gel-column fractionation scheme to the as-synthesized carbon dots samples $[9,20]$. The brighter dots thus harvested promise more and better opportunities for fluorescence bioimaging, especially for uses in vivo to improve the contrast against tissue background. Here we report an experimental validation on the use of these highly fluorescent carbon dots for imaging in mice, with especially a demonstration on the competitive performance of these dots to that of the well-established semiconductor QDs under purposely matching experimental conditions.

\section{Experimental Section}

\section{I. Materials}

Carbon nano-powders, $O, O^{\prime}$-bis(3-aminopropyl) polyethylene glycol $\left(M_{\mathrm{W}} \sim 1,500, \mathrm{PEG}_{1500 \mathrm{~N}}\right)$ and thionyl chloride $(>99 \%)$ were purchased from Aldrich, zinc acetate dihydrate $\left(\mathrm{Zn}\left(\mathrm{OOCCH}_{3}\right)_{2} \bullet 2 \mathrm{H}_{2} \mathrm{O},>98 \%\right)$ and sodium sulfide $\left(\mathrm{Na}_{2} \mathrm{~S} \bullet 9 \mathrm{H}_{2} \mathrm{O},>98 \%\right)$ from Alfa, 
and nitric acid, $\mathrm{N}, \mathrm{N}$-dimethylformamide (DMF, 99\%), and sodium dodecyl sulfate (SDS, 99\%) from VWR. The aqueous CdSe/ZnS quantum dots solution (Qdot ${ }^{\circledR} 525$ ITK $^{\mathrm{TM}}$ amino (PEG) QDs) was acquired from Invitrogen. Millipore Durapore membrane filters (pore size $0.22 \mu \mathrm{m}$ ) and dialysis membrane tubing were supplied by Spectrum Laboratories. Water was deionized and purified by being passed through a Labconco WaterPros water purification system.

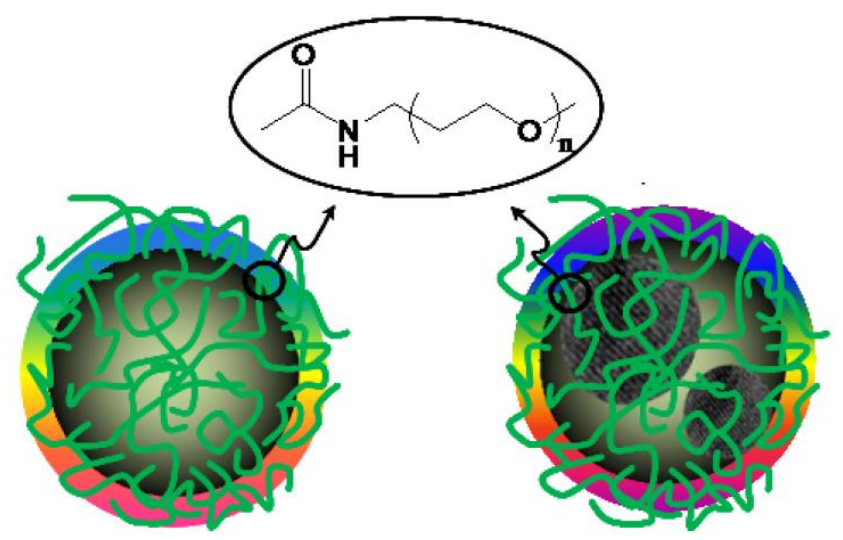

Figure SI. (Scheme I) Cartoon illustrations of non-doped (left) and doped (right) carbon dots [20].

\subsection{Measurement}

Baxter Megafuge (model 2630) and Beckman-Coulter ultracentrifuge (Optima L90K with a type $90 \mathrm{Ti}$ fixed-angle rotor) were used. UV/vis absorption spectra were recorded on a Shimadzu UV2101-PC spectrophotometer. Fluorescence spectra were measured on a Jobin-Yvon emission spectrometer equipped with a $450 \mathrm{~W}$ xenon source, Gemini-180 excitation and Tirax-550 emission monochromators, and a photon counting detector (Hamamatsu R928P PMT at $950 \mathrm{~V})$. Quinine sulfate and 9,10-bis(phenylethynyl)-anthracene were used as standards in the determination of fluorescence quantum yields by the relative method (matching the absorbance at the excitation wavelength between the sample and standard solutions and comparing their corresponding integrated total fluorescence intensities). Atomic force microscopy (AFM) images were acquired in the acoustic AC mode on a Molecular Imaging PicoPlus AFM system equipped with a multipurpose scanner and a NanoWorld point probe $\mathrm{NCH}$ sensor. Transmission electron microscopy (TEM) characterization was carried out on Hitachi 9500 TEM and Hitachi HD-2000 S-TEM systems. Confocal fluorescence images were obtained on a Leica laser scanning confocal fluorescence microscope
(DM IRE2, with Leica TCS SP2 SE scanning system) equipped with an argon ion laser (JDS Uniphase). The fluorescence imaging in mice was performed on a MAG Biosystems Lumazone FA in vivo Imaging System equipped with an Asahi Max-301 xenon arc source for excitation through a liquid light guide and a Princeton Instruments PIXIS:1024B digital CCD camera system as detector. Band-pass and cut-off filters were used for the selection of excitation and emission wavelengths.

\subsection{Carbon Dots}

A carbon nano-powder sample $(2 \mathrm{~g})$ was refluxed in an aqueous nitric acid solution (2.6 M, 200 $\mathrm{mL}$ ) for $12 \mathrm{~h}$. The mixture back at room temperature was dialyzed against fresh water, followed by centrifuging at $1,000 \mathrm{~g}$ to retain the supernatant. The surface-oxidized small carbon nanoparticles recovered from the supernatant $(100 \mathrm{mg})$ were refluxed in neat thionyl chloride for $6 \mathrm{~h}$. Upon the removal of excess thionyl chloride on a rotary evaporator with a vacuum pump, the sample was mixed well with PEG $_{1500 \mathrm{~N}}$ $(1 \mathrm{~g})$ in a flask. The mixture was heated to $110^{\circ} \mathrm{C}$, and vigorously stirred at that temperature under nitrogen protection for 3 days. The sample back at room temperature was dispersed in water, followed by centrifuging at $25,000 \mathrm{~g}$ to retain the supernatant as an aqueous solution of the as-prepared carbon dots. The sample solution was concentrated and then loaded onto a Sephadex G-100 ${ }^{\mathrm{TM}}$ gel-column packed in house for fractionation [9]. Fluorescence quantum yields of the fractions were determined, and those more fluorescent were combined into a single aqueous solution of carbon dots ( $40 \%$ in fluorescence quantum yield) for further characterization and bioimaging.

For the surface-doping with ZnS, the surface-oxidized carbon nanoparticles $(600 \mathrm{mg})$ were dispersed in DMF $(200 \mathrm{~mL})$ via sonication for $30 \mathrm{~min}$, and to the suspension was first added $\mathrm{Zn}\left(\mathrm{OOCCH}_{3}\right)_{2} \bullet 2 \mathrm{H}_{2} \mathrm{O}(680 \mathrm{mg}$, $3.1 \mathrm{mmol})$ under vigorous stirring and then slow dropwise addition of an aqueous $\mathrm{Na}_{2} \mathrm{~S}$ solution $(0.62 \mathrm{M}, 5 \mathrm{~mL})$ at room temperature. The mixture was centrifuged at $3,000 \mathrm{~g}$, and the precipitate was retained and repeatedly washed with distilled water to obtain the ZnS-doped carbon nanoparticles $(881 \mathrm{mg})$.

The ZnS-doped carbon nanoparticles $(200 \mathrm{mg}$ ) were dispersed in an aqueous SDS solution $(1 \mathrm{wt} \%$, $120 \mathrm{~mL}$ ) via sonication for $30 \mathrm{~min}$. Upon filtration, the filter cake was washed repeatedly with water and then dried. The solid sample was mixed thoroughly with PEG $_{1500 \mathrm{~N}}(1.9 \mathrm{~g})$, and the mixture was heated to $110^{\circ} \mathrm{C}$ and stirred at that temperature for $72 \mathrm{~h}$ under nitrogen protection. The reaction mixture back at 
room temperature was dispersed in water, followed by centrifuging at $25,000 \mathrm{~g}$ to retain the supernatant as an aqueous solution of as-prepared $\mathrm{C}_{\mathrm{Zns}}$-Dots. The sample solution was similarly concentrated and then fractionated on the same Sephadex G-100 ${ }^{\mathrm{TM}}$ gel-column [20]. Fluorescence quantum yields of the fractions were determined, and those more fluorescent were combined into a single aqueous solution of $\mathrm{C}_{\mathrm{ZnS}}$-Dots $(60 \%$ in fluorescence quantum yield) for further characterization and bioimaging.

\subsection{Imaging in Mice}

All of the animal experiments were performed at Clemson University by strictly following the IACUC (Institutional Animal Care and Use Committee) approved protocols. Female DBA/1 mice $(\sim 25 \mathrm{~g}$, acquired from Harlan) were housed in plastic cages (three mice/cage) at the Godley-Snell Research Center at Clemson University and kept on a $12 \mathrm{~h}$ light/dark cycle, with food and water provided ad libitum. Following the acclimation of one week, the mice were randomly divided into groups of 3 mice per group for imaging evaluations. Throughout the imaging experiments the mice were kept under anaesthesia (3\% isoflurane flow). Two injection modes (subcutaneous and front extremity) were used for the in vivo imaging. Before subcutaneous injection, the back area surrounding the injection point on the mouse was shaved to minimize autofluorescence. The control group in both injection modes was injected with equivalent volume of phosphate buffered saline (PBS). For the front extremity injection, the mice $24 \mathrm{~h}$ post-injection were sacrificed by $\mathrm{CO}_{2}$ treatment under anaesthesia. The axillary lymph nodes were dissected for fluorescence imaging. All images were processed and analyzed by using the NIH-commissioned and supplied Image J software (http://rsbweb.nih. gov/ij/).

\section{Results and Discussion}

Small carbon nanoparticles (mostly less than 10 $\mathrm{nm}$ in diameter) were harvested from the commercially supplied carbon nano-powders (Aldrich) by first the aqueous nitric acid treatment and then a combination of dialysis to remove impurities and vigorous centrifuging to retain the supernatant. These nanoparticles were functionalized with $\mathrm{PEG}_{1500 \mathrm{~N}}$ in classical amidation reaction to yield PEGylated carbon dots (Scheme 1/Figure S1). The as-prepared sample was fractionated on a gel-column (Sephadex ${ }^{\mathrm{TM}}$ G-100) to harvest the more fluorescent portion of the sample [9], with the corresponding fluorescence quantum yield in the green of $40 \%$ (440 nm excitation, figure 1).
The sample solution was diluted for the preparation of specimens for characterization by microscopy techniques. Both AFM and TEM results showed dot-like images (figure 2), agreeing well with those for carbon dots reported previously [9].

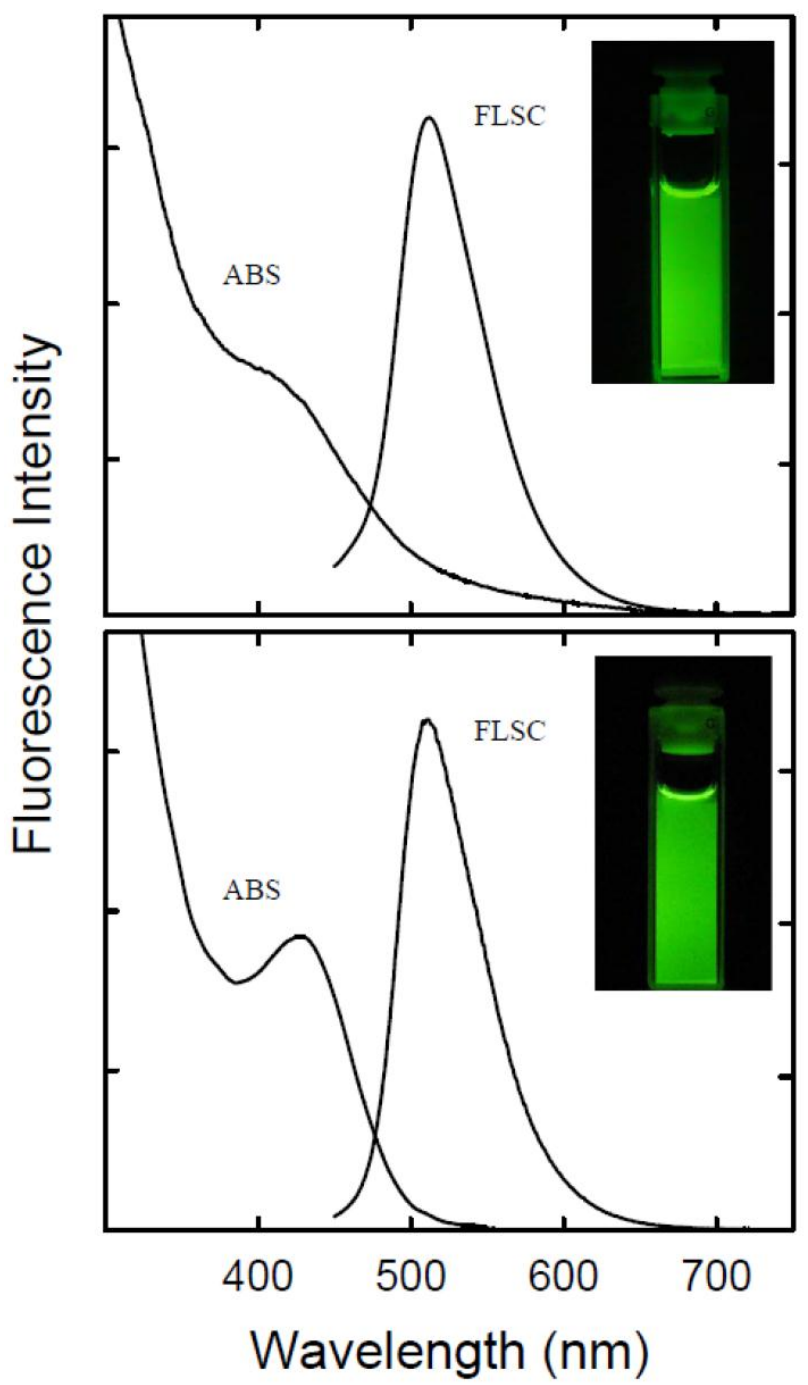

Figure I. Absorption (ABS) and fluorescence (FLSC, $440 \mathrm{~nm}$ excitation) spectra of the carbon dots without (upper) and with the $\mathrm{ZnS}$ doping (lower) in aqueous solutions, with fluorescence quantum yields of $40 \%$ and $60 \%$, respectively. In the insets are photos of the corresponding solutions (excitation at $440 \mathrm{~nm}$ and emissions monitored through a $530 \mathrm{~nm}$ cut-off filter). 

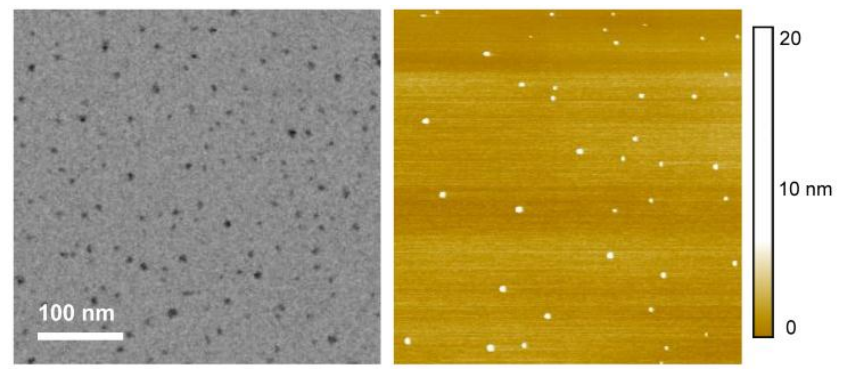

Figure 2. TEM (left) and AFM (right) images of the carbon dots (without $\mathrm{ZnS}$ doping) used in the optical imaging experiments.

The carbon nanoparticles could be suspended in aqueous solution in a relatively stable fashion, thus serving as nucleation centers in the titration of zinc acetate with sodium sulfide for the formation of $\mathrm{ZnS}$ to yield ZnS-doped carbon nanoparticles [24]. The surface-doping level likely varied from particle to particle, so that a surfactant (sodium dodecyl sulfate or SDS)-assisted dispersion procedure in favor of the carbon nanoparticles with more $\mathrm{ZnS}$ doping was used to exclude those with no or a negligible level of doping. Subsequently, those nanoparticles with the surface completely covered by ZnS (thus no accessible carboxylic acid moieties on the particle surface for reactions with amino molecules) were discriminated in the functionalization chemistry with PEG $_{1500 \mathrm{~N}}$ [20]. The functionalization reaction conditions were similar to those used for carbon dots without the surface doping, resulting in ZnS-doped carbon dots (denoted

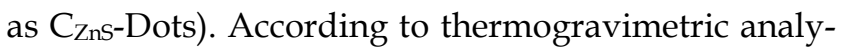
sis (TGA), the estimated core composition in terms of C:ZnS molar ratio in the $C_{Z n s}$-Dots was about 20:1. The as-prepared sample was similarly fractionated on the gel-column to harvest more fluorescent $\mathrm{C}_{\mathrm{Zns}}$-Dots [20], with the observed fluorescence quantum yield in the green of $60 \%$ (440 nm excitation, figure 1). These dots were diluted for the preparation of specimens for characterization by AFM and TEM, and the representative results are shown in figure 3 . The vigorously diluted solution was also used to disperse the dots on cover-glass surface for confocal fluorescence imaging under the same specimen and measurement conditions as those for commercially supplied CdSe/ZnS QDs (Invitrogen Qdot ${ }^{\circledR} 525$ ITK $^{\mathrm{TM}}$ amino (PEG) QDs, fluorescence quantum yield $\sim 60 \%$ verified). As shown in figure 4 , the well-dispersed $\mathrm{C}_{\mathrm{Zns}}$-Dots and the CdSe/ZnS QDs are quite similar in fluorescence brightness at essentially the individual dot level.
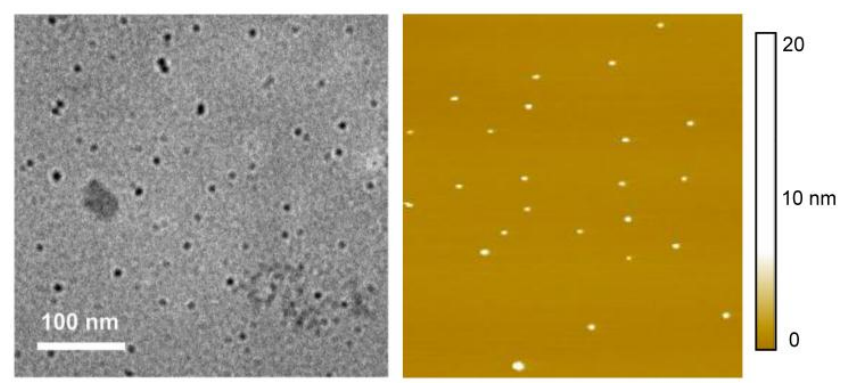

Figure 3. Representative TEM (left) and AFM (right) images of the

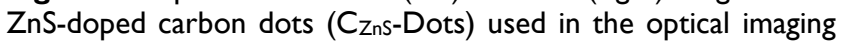
experiments.

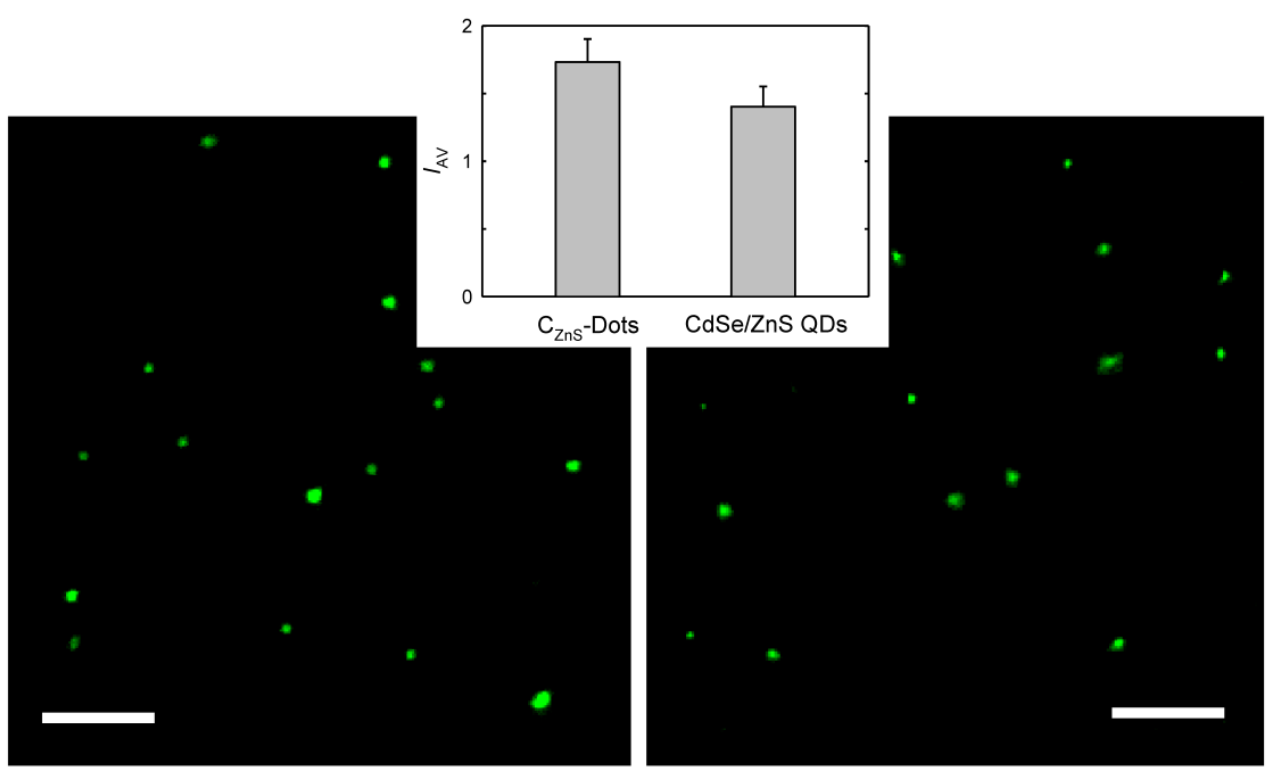

Figure 4. Confocal fluorescence images ( $458 \mathrm{~nm}$ excitation, $10 \mu \mathrm{m}$ for the scale bars) of the CZns-Dots (left) and the commercially supplied CdSe/ZnS QDs (right). In the inset is a comparison on the average image intensity $\left(l_{\mathrm{AV}}\right)$ for 60 dots between the two specimens. 
The highly fluorescent carbon dots without and with the $\mathrm{ZnS}$ doping (quantum yields $40 \%$ and $60 \%$, respectively) were evaluated in terms of fluorescence imaging in mice [23]. The animal experiments were performed by strictly following the IACUC (Institutional Animal Care and Use Committee) approved protocols. Female DBA/1 mice ( $25 \mathrm{~g})$ were housed in plastic cages (three mice/cage) at the Godley-Snell Research Center for animal research at Clemson University on a $12 \mathrm{~h}$ light/dark cycle and provided with food and water ad libitum. Following the acclimation of one week, the mice were randomly divided into groups of three mice per group. For the injection and subsequent imaging, the mice were kept under anesthesia with $3 \%$ isoflurane flow.

Before the subcutaneous injection, the back area surrounding the injection point on the mouse was shaved to avoid autofluorescence. The injection volume was kept at $20 \mu \mathrm{L}$, with the solution concentration adjusted to match the targeted optical density at the excitation wavelength. As shown in figure 5, fluorescence emissions from the subcutaneously injected dots could readily be detected in a Lumazone FA in vivo imaging system (MAG Biosystems), with a relatively shorter fluorescence collection time and improved imaging contrast in comparison with those in the use of as-prepared less fluorescent carbon dots [23]. The image brightness was obviously higher for the $\mathrm{C}_{\mathrm{Zns}}$-Dots sample, consistent with the corresponding higher fluorescence quantum yield in solution. In mice the subcutaneously injected carbon dots diffused relatively slowly, with the gradual fading of fluorescence signals in about $24 \mathrm{~h}$ post-injection.
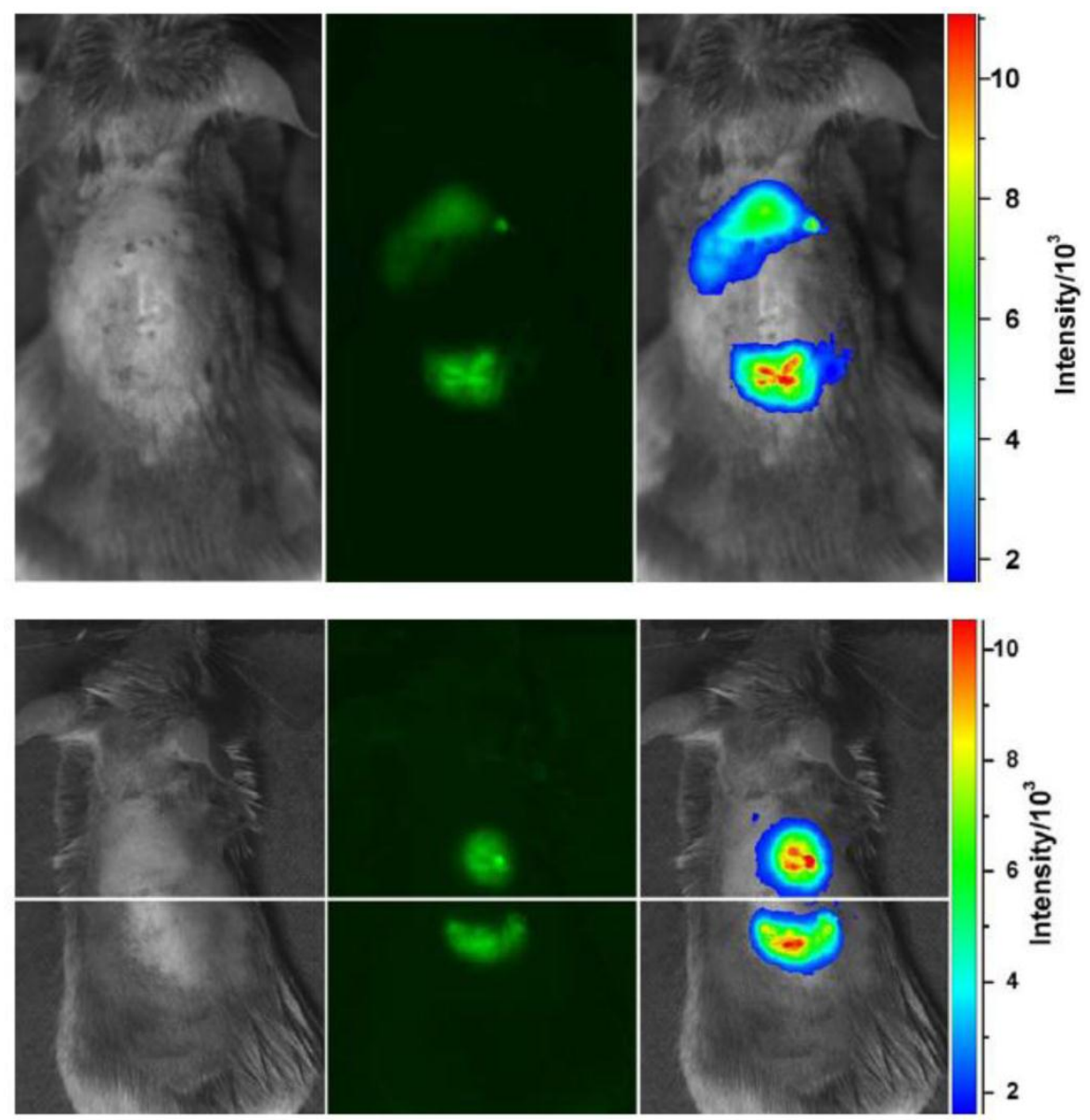

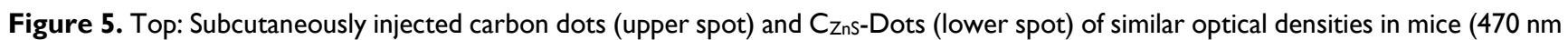
and $525 \mathrm{~nm}$ band-pass filters for excitation and emission, respectively). Bottom: Subcutaneously injected C Zns-Dots (upper spot) and Invitrogen Qdot ${ }^{\circledR} 525$ ITKTM amino (PEG) QDs (lower spot) of similar optical densities in mice (434 nm band-pass filter for excitation and $474 \mathrm{~nm}$ cut-off filter for emission collection). The color-coded images (right) were processed with Image from $\mathrm{NIH}$. 
In a comparison of fluorescence imaging performance with commercially supplied CdSe/ZnS QDs (Invitrogen Qdot ${ }^{\circledR} 525$ ITK ${ }^{\mathrm{TM}}$ amino (PEG) QDs), the concentration of the subcutaneously injected QDs was adjusted such that the optical density was approximately the same as that of the carbon dots in vivo. The resulting fluorescence images for $\mathrm{C}_{\mathrm{Zns}}$-Dots and the CdSe/ZnS QDs in mice were of similar brightness (figure 5), consistent with their similar fluorescence quantum yields in solution and comparable fluorescence imaging results at the individual dot level (figure 4).

For potential uses of carbon dots for fluorescence imaging in vivo, a relatively straightforward demonstration is on tracking the migration of the dots through lymph vessels in mice following the paw injection $[23,25,26]$. For the highly fluorescent carbon dots without and with $\mathrm{ZnS}$ doping (quantum yields $40 \%$ and $60 \%$, respectively), they could both migrate along the arm upon their intradermal injection into the front extremity (figure 6). However, the migration of the $\mathrm{C}_{\mathrm{Zns}}$-Dots was apparently less pronounced, with the relative brightness in the fluorescence images (figure 6) of the migrated dots suggesting less $\mathrm{C}_{\mathrm{ZnS}}$-Dots (more fluorescent) migrated. The axillary lymph nodes were harvested and dissected at $24 \mathrm{~h}$ post-injection, where strong fluorescence emissions from the carbon dots were observed (figure 6), further confirming the migration of the dots and the preservation of their fluorescence properties in vivo.

The improved optical imaging performance of these more fluorescent carbon dots in mice further confirms that the excellent fluorescence properties of carbon dots observed in solutions and on surface at the individual dot level are preserved in vivo, suggesting significant application potentials of carbon dots. Particularly encouraging is the fact that the in vivo performance of the carbon dots is competitive to that of the well-established CdSe/ZnS QDs, beyond the obvious advantage of carbon dots being nontoxic according to available experimental evaluations $[10,11,21,27-29]$. The results from this study justify the further development of carbon dots into a new class of high-performance yet benign contrast agents for optical bioimaging.
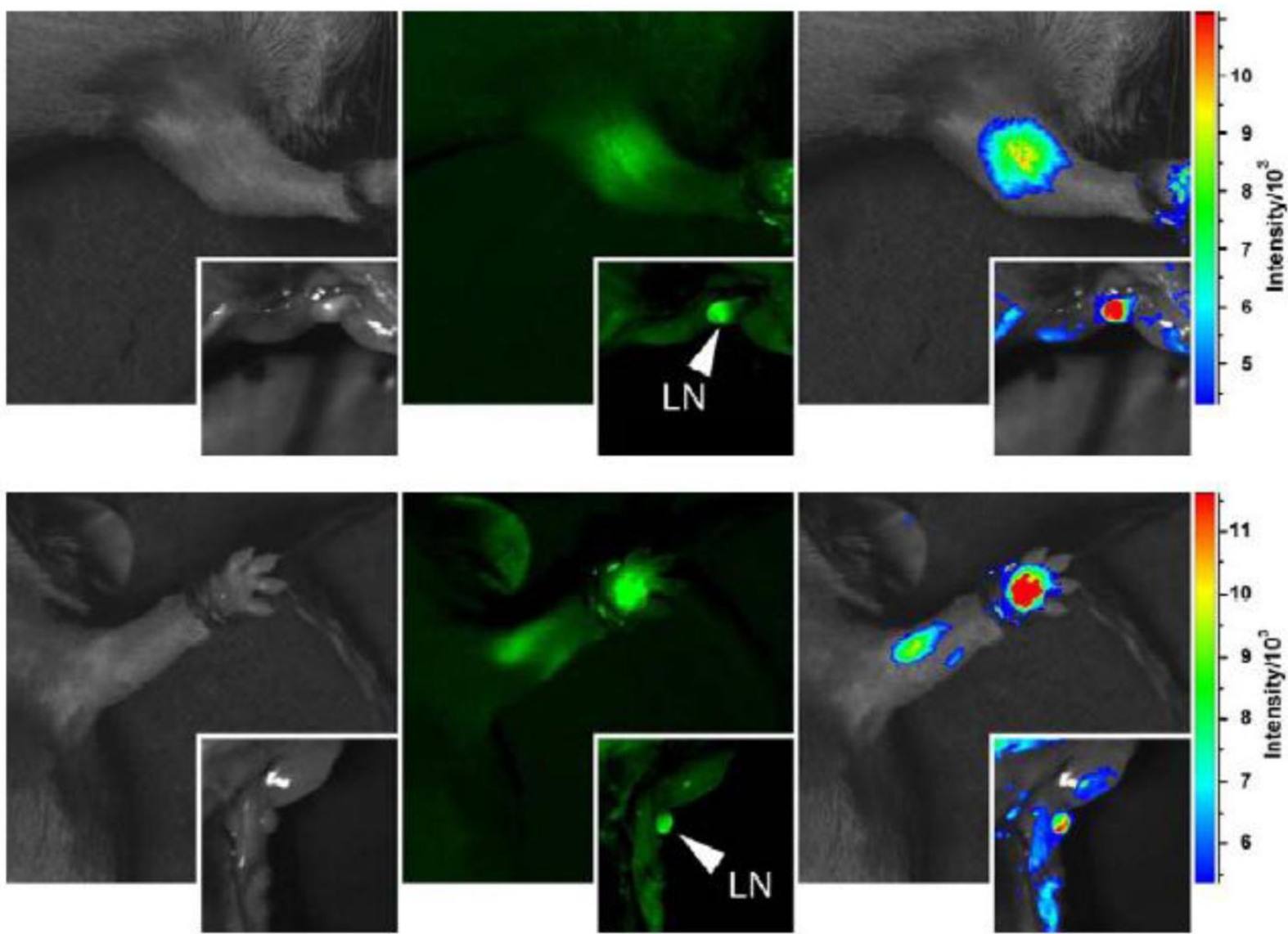

Figure 6. Intradermally injected carbon dots (upper) and CZns-Dots (lower) of similar optical densities in mice, with images of dissected lymph nodes in the insets (470 $\mathrm{nm}$ and $525 \mathrm{~nm}$ band-pass filters for excitation and emission, respectively). The color-coded images (right) were processed with Imagej from $\mathrm{NIH}$. 


\section{Acknowledgment}

This work was initiated and supported by funding from NIH and then from NSF. L.C. was supported by a Susan G. Komen for the Cure Postdoctoral Fellowship. S.S. was a participant supported by Palmetto Academy, an education-training program managed by South Carolina Space Grant Consortium.

\section{Conflict of Interest}

The authors have declared that no conflict of interest exists.

\section{References}

1. Sun Y-P, Zhou B, Lin Y, et al. Quantum-sized carbon dots for bright and colorful photoluminescence. J. Am. Chem. Soc 2006; 128: 77561.

2. Cao L, Wang $X$, Meziani MJ, et al. Carbon dots for multiphoton bioimaging. J. Am. Chem. Soc 2007; 129: 11318-11319.

3. Bourlinos AB, Stassinopoulos A, Anglos D, et al. Surface functionalized carbogenic quantum dots. Small 2008; 4: 455-458.

4. Bourlinos AB, Stassinopoulos A, Anglos D, et al. Photoluminescent carbogenic dots. Chem. Mater 2008; 20: 4539-4541.

5. Tian L, Ghosh D, Chen W, et al. Nanosized carbon particles from natural gas soot. Chem. Mater 2009; 21: 2803-2809.

6. Hu S-L, Niu K-Y, Sun J, et al. One-step synthesis of fluorescent carbon nanoparticles by laser irradiation. J. Mater. Chem 2009; 19: 484-488.

7. Peng H, Travas-Sejdic J. Simple aqueous solution route to luminescent carbogenic dots from carbohydrates. Chem. Mater 2009; 21: 5563-5565.

8. Jiang HQ, Chen F, Kagally MG, Denes FS. New strategy for synthesis and functionalization of carbon nanoparticles. Langmuir 2010; 26: 1991-1995.

9. Wang X, Cao L, Yang S-T, et al. Bandgap-like strong fluorescence in functionalized carbon nanoparticles. Angew Chem. Int. Ed 2010; 49: 5310-5314.

10. Li Q, Ohulchanskyy TY, Liu RL, et al. Photoluminescent carbon dots as biocompatible nanoprobes for targeting cancer cells in vitro. J. Phys. Chem. C 2010; 114: 12062-12068.

11. Qiao Z-A, Wang Y, Gao Y, et al. Commercially activated carbon as the source for producing multicolor photoluminescent carbon dots by chemical oxidation. Chem. Commun 2010; 46: 8812-8814.

12. Li H, He X, Liu Y, et al. One-step ultrasonic synthesis of water-soluble carbon nanoparticles with excellent photoluminescent properties. Carbon 2011; 49: 605-609.

13. Li H, He X, Liu Y, et al. Synthesis of fluorescent carbon nanoparticles directly from active carbon via a one-step ultrasonic treatment. Mater. Res. Bull 2011; 46: 147-151.

14. Li X, Wang H, Shimizu Y, et al. Preparation of carbon quantum dots with tunable photoluminescence by rapid laser passivation in ordinary organic solvents. Chem. Commun 2011; 47: 932-934.

15. Wang F, Pang S, Wang L, et al. One-step synthesis of highly luminescent carbon dots in noncoordinating solvents. Chem. Mater 2010; 22: 4528-4530.

16. Alivisatos AP. Semiconductor clusters, nanocrystals, and quantum dots. Science 1996; 271: 933-937.

17. Bruchez M, Moronne M, Gin P, et al. Semiconductor nanocrystals as fluorescent biological labels. Science 1998; 281: 2013-2016.

18. Wu X, Liu H, Liu J, et al. Immunofluorescent labeling of cancer marker Her2 and other cellular targets with semiconductor quantum dots. Nat. Biotechnol 2003; 21: 41-46.
19. Resch-Genger U, Grabolle M, Cavaliere-Jaricot S, et al. Quantum dots versus organic dyes as fluorescent labels. Nat. Methods 2008; 5: 763-775.

20. Anilkumar P, Wang X, Cao L, et al. Toward quantitatively fluorescent carbon-based "quantum" dots. Nanoscale 2011; 3: 2023-2027.

21. Yang S-T, Wang X, Wang H, et al. Carbon dots as nontoxic and high-performance fluorescence imaging agents. J. Phys. Chem. C 2009; 113: 18110-18114.

22. Liu R, Wu D, Liu S, et al. An aqueous route to multicolor photoluminescent carbon dots using silica spheres as carriers. Angew. Chem. Int. Ed 2009; 48: 4598-4601.

23. Yang S-T, Cao L, Luo P, et al. Carbon dots for optical imaging in vivo. J. Am. Chem. Soc 2009; 131: 11308-11309.

24. Sun Y-P, Wang X, Lu F, et al. Doped carbon nanoparticles as a new platform for highly photoluminescent dots. J. Phys. Chem. C 2008; 112: 18295-18298.

25. Kim S, Lim YT, Soltesz EG, et al. Near-infrared fluorescent type II quantum dots for sentinel lymph node mapping. Nat. Biotechnol 2004; 22: 93-97.

26. Ballou B, Ernst LA, Andreko S, et al. Sentinel lymph node imaging using quantum dots in mouse tumor models. Bioconjug Chem 2007; 18: 389-396.

27. Zhao Q-L, Zhang Z-L, Huang B-H, et al. Facile preparation of low cytotoxicity fluorescent carbon nanocrystals by electrooxidation of graphite. Chem. Commun 2008;: 5116-5118.

28. Ray SC, Saha A, Jana NR, Sarkar R. Fluorescent carbon nanoparticles: Synthesis, characterization, and bioimaging application. J. Phys. Chem. C 2009; 113: 18546-18551.

29. Liu J-H, Anilkumar P, Cao L, et al. Cytotoxicity evaluations of fluorescent carbon nanoparticles. Nano Life 2010; 1: 153-161. 